\title{
- Review on Constipation in Adults
}

\section{Monali P. Walke ${ }^{1}$, Sheetal Sakharkar ${ }^{2}$}

\section{IJCRR}

Section: Healthcare ISI Impact Factor (2019-20): 1.628

IC Value (2019): 90.81 $\operatorname{SJIF}(2020)=7.893$

(c) (i) (8)

Copyright@IJCRR
'MSc. Nursing student, Dept. of Medical-surgical Nursing, Smt. Radhikabai Meghe Memorial College of Nursing, Datta Meghe Institute of Medical Sciences (Deemed to be University) Sawangi (M), Wardha, Maharashtra, India; ${ }^{2}$ Lecturer Dept. of Medical-Surgical Nursing, Smt. Radhikabai Meghe Memorial College of Nursing, Datta Meghe Institute of Medical Sciences (Deemed to be University) Sawangi (M), Wardha, Maharashtra, India.

\section{ABSTRACT}

Constipation is a gastrointestinal tract condition that can lead to abnormal stools, uncomfortable storage and passing with pain and stiffness. Acute constipation may required intestines to close, and that may also require surgery. Constipation occurs due to improper diet, Diseases conditions can causing secondary reason forgot constipation. Prevalence of constipation $16 \%$ in adult worldwide (ranging from $0.7 \%$ to $79 \%$ ); Prevalence present in adults 60 to 110 years of age at $33.5 \%$. Great Britain given opinion which is based on the study conducted on healthy person concluded that three bowel movements per week and three bowel movements per day, $99 \%$ of the population an accurate evaluation of the symptom must provide an aware and guided background of the relevant characteristics. Initial history and physical examination, rectal examination, Endoscopy, Anorectal manometry, Barium enema, Colonic transit study are important tools for the diagnosis of Constipation. Pharmacological treatment Bulk-Forming Agents, Stimulants, osmotic agents, Stool Softeners and Emollients, Traditional Chinese Medicine and last one is surgical treatment is given to client for relieving constipation. Abnormal stools, uncomfortable storage and passing with pain and stiffness occurred in constipation. There are lots of risk factor responsible for getting constipation like diet, disease condition etc. For treatment need to medication and also surgical treatment is also available for treating constipation.

Key Words: Constipation, Cause, Diagnosis of constipation, Pharmacological treatment

\section{INTRODUCTION}

Constipation is a condition of the gastrointestinal tract which can lead to abnormal stools, uncomfortable storage passage with the feeling of stiffness and pain. Acute constipation may cause a close of the intestine, and that also need to conduct surgery. ${ }^{1}$ There is no any till ideal constipation definition. Therefore, the main initial approaches can be considered in history and physical examination. By given selfly -reporting constipation and with the help of the formal parameters, several meanings are defined. Other definitions of chronic constipation have been considering in a scientific way like secondary causes like medication, systemic diseases or may be neurological. It is deemed idiopathic or may primarily cause constipation. ${ }^{2}$

Pathogenesis is multifactor which can put the effect of genetic predisposition, lack of sufficient fluid intake capacity, decreased mobilization, socioeconomic status, disruption of the hormonal balance, side effects of medicinal products. Constipation is a chronic basically issue of the gastrointesti- nal tract that causes several community expenditures with an approximate incidence of $1 \%$ to $80 \%$, where the disease is distinguished by broad regional variability. It may be led to a broad prevalence of constipation rates. ${ }^{3-5}$

\section{DEFINITION}

Constipation is not a disorder, just a symptom. Constipation is more generally regarded as infrequent bowel movements, typically less than 3 stools a week. Nevertheless, individuals may have other grievances, including:

- Straining of intestinal movements

- Excessive time required for a bowel movement to pass

- Rough stools

- Pressure with intestinal movements secondary to strain

- Pain in the abdomen

- Bloating abdominally.

- The feeling of an incomplete evacuation of the intestines.

\section{Corresponding Author:}

Ms. Monali P. Walke, MSc. Nursing student, Department of Medical-surgical Nursing, Smt. Radhikabai Meghe Memorial College of Nursing, Datta Meghe Institute of Medical Sciences (Deemed to be University) Sawangi (M), Wardha, Maharashtra, India; Email: Monaliwalke9764@, gmail.com

ISSN: 2231-2196 (Print)

ISSN: 0975-5241 (Online)

Received: 24.09 .2020

Revised: 14.11 .2020

Accepted: 07.01.2021

Published: 19.05 .2021 


\section{PREVALENCE AND EPIDEMIOLOGY}

In studies, constipation is described differently, most of the questionnaire-based research study will result from the organic condition. $16 \%$ of adult constipation (ranging from $0.7 \%$ to $79 \%$ ) worldwide prevalence of constipation. Prevalence was attributed to 33.5 per cent in adults aged 60 to 110 years). ${ }^{5}$ This state is heterogeneous, associated with client quality of life and health care facility use. Iran has a constipation rate of 1.4 per cent to 37 per cent. Age refers to the high prevalence of chronic constipation. ${ }^{6-8}$

\section{EPIDEMIOLOGY OF CONSTIPATION}

Great Britain's opinion on health individuals reported that 99 $\%$ of the population had three bowel movements in a week and three bowel movements in a day. ${ }^{7} 52 \%$ of constipation was described as straining revealed by Sandler and Dross man to move through faecal material. In the survey conducted on young adults who do not seek medical attention, the phase of going through hard stools was felt by $44 \%$, it was only the uncommon movement of stools felt by $32 \%$, and 34 $\%$ were unable to pass.

\section{RISK FACTOR}

The following are factors that may increase your risk of chronic constipation:

- Be an older adult

- Being a woman

- Dehydrated being

- Eating a diet deficient in fibre

- Getting little or no physical activity

- Taking certain medications, including sedatives, opioid pain medications, some antidepressants

- Take certain drugs, including sedatives, opioid pain medications, certain antidepressants, or blood pressure control medications

- Having a mental health condition including anxiety or an eating disorder ${ }^{8}$

\section{CAUSES}

Constipation most often happens when waste or stool passes too slowly or can not be easily removed from the rectum via the digestive tract, which can cause the stool to become harsh and dry. Many potential causes of chronic constipation. ${ }^{10}$

\section{Diet}

A low-fibre diet, low fluid intake, or dieting can cause or worsen constipation. Dietary fibre helps minimise colonic transport time, raises the bulk of stool but softens stool at the same time. Diets low in fibre, therefore, can contribute to primary constipation. ${ }^{9}$

\section{Medications}

Many drugs have side effects of constipation. Some include opioids, diuretics, antidepressants, antihistamines, antispasmodics, anticonvulsants, tricyclic antidepressants, antiarrhythmics, beta-adrenoceptor antagonists, antidiarrheals, ondansetron-like 5-HT3 receptor antagonists, and aluminium antacids.

\section{Medical conditions}

Metabolic and endocrine disorders that may lead to constipation include hypercalcemia, hypothyroidism, hyperparathyroidism, porphyria, chronic kidney disease, panhypopituitarism, diabetes mellitus and cystic fibrosis. Constipation is normal in people with muscle and myotonic dystrophy, too.

\section{Psychological}

A recurrent cause of constipation is the voluntary withholding of the stool. The choice of withholding may be attributed to reasons such as pain fear, public bathroom fear, or laziness. A mixture of reinforcement, water, fibre, and laxatives can be effective in solving the issue when a child keeps in the stool.

\section{Congenital}

A variety of birth-related illnesses may cause constipation in children. As a group, they are rare, the most common being Hirschsprung's disease (HD). Congenital structural defects can also lead to constipation, including anterior anus displacement, anus imperforation, strictures, and small left colon syndrome. ${ }^{10}$

\section{Blockages in the colon or rectum}

- Blockages may delay or interrupt the passage of the stool in the colon or rectum. Includes causes:

- Narrow skin tears around the anus (anal fissure)

- A blockage (bowel obstruction) in the intestines

- Colon carcinoma ${ }^{11}$

\section{Problems with the nerves around the colon and rectum}

- The nerves that cause muscles in the colon and rectum to contract and pass stool through the gut may be affected by neurological disorders. Includes causes:

- Damage to body working nerves (autonomic neuropathy);

- Multiple Sclerosis

- Parkinson's illness ${ }^{12}$ 


\section{Difficulty with the muscles involved in the elimination}

- Pelvic muscle problems that require bowel movement can cause chronic constipation. Such issues can involve:

- Impossibility of relaxing pelvic muscles to facilitate bowel movement (anism)

- Pelvic muscles, which do not adequately manage relaxation and contraction (dyssynergia). ${ }^{13}$

\section{Conditions that affect hormones in the body}

In your body, hormones help regulate fluids. Constipation can result from diseases and conditions that upset the hormone balance, including:

- Parathyroid gland overactive (hyperparathyroidism);

- Breastfeeding

- Underactive (hypothyroidism) thyroid ${ }^{14}$

\section{PATHOPHYSIOLOGY}

Although the frequency of bowel movements is mostly concentrated on in the definition of constipation, consumers can have a wide variety of concerns. The frequently cited lower limit of appropriate stool frequency is three per week and two or fewer stools per week can be used as one of the Rome criteria. The frequency was only one out of six in this summary (including straining, hard stools, and a feeling of incomplete evacuation). Symptoms have been described as straining (decreasingly significant), painfully hard stools, unproductive urges, infrequency and a feeling of incomplete evacuation in patient records..$^{15}$

\section{SYMPTOMS}

The main symptoms of constipation are:

- Difficulty passing stool

- Straining when passing stool

- Passing less stool than usual

- Lumpy, dry, or hard stool

- Passage of liquid faecal seepage

- Infrequent passage of stool

- Frequent but non productive desire to defecate

- Straining at stools

- Nausea and vomiting

- Anorexia

- Abdominal distention

- Dull headache

- Pain with defecation

- A loss of appetite ${ }^{16}$

\section{DIAGNOSTIC EVALUATION}

Variables may be identified for clinical evaluation like stool frequency, consistency, symptom intensity, prolonged obstruction and history of avoiding a defecation call, and feeling of insufficient evacuation. To determine common constipation signs (alarming signs), medical history and environmental causes and also medicines. ${ }^{17,18}$

Endoscopy: Procedures such as flexible sigmoidoscopy (FS) or colonoscopy are useful by using chronic laxative and mucous lesions to obtain evidence of the cause of unexplained symptoms. ${ }^{17}$

Anorectal manometry: It is a diagnostic tool for evaluating the activity of anurectomal strain, which can also show rectal compliance with rectal reflexes, rectal sensation etc.

Balloon expulsion testing: It is used for the measurement of dssynergic defecation in addition to anorectal manometry. In addition, BET was administered as part of pelvic floor dyssynergy identification or to separate patients with constipation without pelvic dyssynergy. ${ }^{18}$

Barium enema: It is a colon X-ray technique for evaluating improvements or structural defects of the colon filled with contrast like metallic material (barium).

Colonic transit study: For recording, a client may need to swallow a capsule with either a radiopaque marker or a wireless device. The movement of the capsule through the colon will be documented on X-rays for several days. ${ }^{19}$

\section{COMPLICATION}

1. Faecal incontinence: Overflow through moving through isolated obstructing bolus may cause fresh faecal matter to complicate the diagnosis of chronic constipation unless a rectal examination has been performed.

2. Haemorrhoids: In the anorectal junction plexus and anterior venous anastomoses, excessive stress and intra-abdominal pressure raise venous pressure.

3. Anal fissure: During the evacuation of hard stool, trauma and sudden tearing of the anal mucosa is an indicator of the situation, but the internal anal sphincter spasm is assumed to be the perpetuating cause that triggers the relative ischemia. ${ }^{20}$

\section{PHARMACOLOGICAL TREATMENT}

Chronic constipation medications can be classified as bulk forming agents, stool softeners and emollients, osmotic agents, stimulants, chloride channel activators.

Bulk-Forming Agents: This agent is used in fibre supplements. With the water, these agents can be extended to increase the bulk of the stool and also to increase bowel movements. E.g. Ex. Psyllium (ispaghula husks), polycarbophil of calcium, methylcellulose. 
Stool Softeners and Emollients: Usually used stool softener such as Sodium docusate that has a deterrent effect. There is no other known research that compares its independent efficacy with placebo; one double-blind randomised trial showed docusate in chronic constipation was lower than psyllium.

Osmotic Agents: Because of its hyperosmolar existence, agents such as polyethene glycol (PEG), lactulose, sorbitol, and magnesium hydroxide often absorb and retain water and promote stool movement. The stools increase in softness and thickness.

Stimulants: Stimulants work on the colon's myenteric plexus and boost peristaltic contractions. They also elevate water absorption from the lumens. Ex. Senna, Bisacodyl

Chloride Channel Activators: These are essentially the bicyclic fatty acids that can help activate chloride from 2 channels to increase the secretion of intestinal fluid in the apical gastrointestinal epithelium membrane. ${ }^{20,21}$

Other Treatments: Lactobacillus and Bifid bacteria should be considered to be associated with harmful pathogens that are bound to the enterocyte surface and then protect the mucosa as symbiotic flora present in the large intestine. These probiotic bacteria are used for the treatment of chronic constipation and other inflammatory bowel diseases. A retrospective study has confirmed that bifid bacterium supplementation can be used for the treatment of dietary hypocaloric constipation.

\section{SURGICAL TREATMENT}

If surgical treatment has failed in constipated patients and the use of colon through mechanical emptying can also be administered to clients with slow bowel movements using an enema system as an alternative to treating patients. Colectomy with ileorectal anastomosis can be used in patients with refractory sluggish transit constipation as a treatment option. ${ }^{21}$

\section{NURSING MANAGEMENT}

- Encourage the client to take 2000 to $3000 \mathrm{~mL} /$ Day of fluid, if not medically contraindicated.

- Enable the client to eat at least $20 \mathrm{~g}$ of dietary fibre a day (e.g. raw fruits, fresh vegetables, whole grains)

- Regular exercise and activity

\section{NURSING DIAGNOSIS}

- Constipation related to pain on defecation

- Constipation related inactivity

- Constipation related decreased dietary intake

\section{DISCUSSION}

Constipation is a condition of the gastrointestinal tract which can lead to abnormal stools, uncomfortable storage passage with the feeling of stiffness and pain. Acute constipation may cause the closure of the intestines, and that also need to conduct surgery. $24.2 \%$ is the prevalence of functional constipation. Age is associated with a High prevalence of chronic constipation. There are many risk factors are can be discussed which can cause constipation like Age and gender distribution, medication, diet, Disorders. Diagnostic evaluation, complication and its management included in this review.

\section{CONCLUSION}

In the general population, the prevalence is $16 \%$ (Range between $0.7 \%$ and $79 \%$ ). Clearly understanding the pathophysiology of chronic constipation and information regarding the pharmacological agent's efficacy and safety can help physicians treat and manage constipation symptoms. The efficacy of various treatment methods has been given. Substantial frustration in patients does not have to be avoided. It comes to mind that who required for identifying the components in their terms. Complete history, full physical, digital rectal evaluation and detailed evaluation. Lubiprostone and linaclotide can be considered as two beneficial medications if the effects are not minimized by the laxatives. It is worth noting that biofeedback therapy is a valuable tool to boost symptoms of bowel and dyssynergic defecation. When medical care of constipated patients has failed, surgical procedures can be implemented.

\section{ACKNOWLEDGEMENT}

Authors acknowledge the immense help received from Principal Dr. Seema Sing, Dept. Of MSN,SRMMCON. Sawangi (M), Wardha, Author also thankful Ms. Ranjana Sharma HOD of dept. of MSN, Sawangi (Meghe), Author also Thankful Guide Ms. Sheetal Sakharkar, Clinical Instructor, Dept. Of MSN, Sawangi (Meghe), for her guidance. The author acknowledges the immense help received from the scholars whose articles are cited and included in references of this manuscript. The authors are also grateful to authors/ editors/publishers of all those articles, journals and books from where the literature for this article has been reviewed and discussed."

\section{Conflict of interest: None}

Financial support: Self 


\section{REFERENCES}

1. Bronner F. Nutritional Aspects and Clinical Management of Chronic Disorders and Diseases. CRC Press; 2002.Vol 1.352

2. Camilleri M, Thompson WG, Fleshman JW, Pemberton JH. Clinical management of intractable constipation. Ann Intern Med 1994;121(7):520-528.

3. FORCE T. Guidelines for the diagnosis and therapy of the vein and lymphatic disorders. Int Angiol 2005;21(2):68-107.

4. Abbott RD, Petrovitch H, White LR, Masaki KH, Tanner CM, Curb JD, et al. Frequency of bowel movements and the future risk of Parkinson's disease. Neurology 2001;57(3):456-462.

5. Schnabel L, Buscail C, Sabate J-M, Bouchoucha M, Kesse-Guyot E, Allès B, et al. Association between ultra-processed food consumption and functional gastrointestinal disorders: Results from the French NutriNet-Santé cohort. Am J Gastroenterol 2018;113(8):1217-1228.

6. Cutler DM, Ghosh K, Messer KL, Raghunathan TE, Stewart ST, Rosen AB. Explaining the slowdown in medical spending growth among the elderly, 19992012. Health Aff (Millwood) 2019;38(2):222-229.

7. Walter S, Hallböök O, Gotthard R, Bergmark M, Sjödahl R. A population-based study on bowel habits in a Swedish community: prevalence of faecal incontinence and constipation. Scand J Gastroenterol 2002;37(8):911-916.

8. Pearce J, Hunter JO. Nutrition and the Gastrointestinal Tract for Athletes. Sport Exerc Nutr. 2011;264-280.

9. Gant SG. Diseases of the Rectum, Anus, and Colon: Including the Ileocolic Angle, Appendix, Colon, Sigmoid Flexure, Rectum, Anus, Buttocks, and Sacrococcygeal Region, C by Samuel Goodwin Gant With 1128 Illustrations on 1085 Figures and 10 Insets in Colors Saunders Company; 1923.

10. de Lorijn F, Reitsma JB, Voskuijl WP, Aronson DC, Fiebo J, Smets AM, et al. Diagnosis of Hirschsprung's disease: a prospective, comparative accuracy study of common tests. J Pediatr 2005;146(6):787-792.
11. Rees PM, Fowler CJ, Maas CP. Sexual function in men and women with neurological disorders. Lancet 2007;369(9560):512-525.

12. Catto-Smith AG. 5. Constipation and toileting issues in children. Med J Aust 2005;182(5):242-246.

13. Albain KS, Barlow WE, Shak S. Prognostic and predictive value of the 21-gene recurrence score assay in postmenopausal women with nodepositive, oestrogenreceptor-positive breast cancer on chemotherapy: a retrospective analysis of a andomised trial. Lancet Oncol 2010;11:55-65.

14. Gordon JS. The Effectiveness of Reflexology as a Adjunct to Standard Treatment in Childhood Idiopathic Constipation A Single Blind Randomised Controlled Trial [PhD Thesis]. Edinburgh Napier University; 2007.

15. Whorton JC. Inner hygiene: constipation and the pursuit of health in modern society. Oxford University Press, USA; 2000.

16. Avila-Rencoret FB. A Novel Endoscopic ScanningProbe Device for Rapid Screening of Gastrointestinal Dysplasia [ $\mathrm{PhD}$ Thesis]. Imperial College London; 2014.

17. Lam TJ. Clinical approach to anorectal disorders. 2015; Thesis. Research VU University Amsterdam, graduation VU University Amsterdam. unknown (vumc.nl)

18. Kathpalia H, Sharma K, Doshi G. Recent trends in Hard Gelatin capsule delivery System. J Adv Pharm Educ Res 2014;4(2).

19. Andrews GR. Diarrhoea and constipation in geriatric practice. Cambridge University Press; 1999:152-158.

20. Bao HF, Liu L, Self J, Duke BJ, Ueno R, Eaton DC. A synthetic prostone activates apical chloride channels in A6 epithelial cells. Am J Physiol-Gastrointest Liver Physiol. 2008;7(3):172-6.

21. Wallis M, McKenzie S, Gyett S, Rayner K, Ellem F, Gass E, et al. Help patients win the constipation battle: Best practice in the prevention and treatment of constipation in adults under 65 years. J Adv Pharm Educ Res 2003;4(5):23-6. 